\title{
掺硅对封闭碳纳米管尖端几何及电子结构影响的 第一原理研究
}

\author{
周俊哲 ${ }^{\circledR}$ 王崇愚 ${ }^{(1)}$ \\ (1) 清华大学物理系, 北京 100084; (2) 中国科学院国际材料物理中心, 沈阳 110016. E-mail: cywang@mail.tsinghua.edu.cn)
}

\begin{abstract}
摘要 采用第一原理 DMol 方法研究了碳纳米管(CNT)尖端替代式掺 Si 对于几何结构及电子结构的影 响. 计算结果表明掺 $\mathrm{Si}$ 引入掺杂态, 使管端整体局域态密度在费米能级附近有很大提高, 在管端形成利 于电荷积聚的原子尺度尖区, 且发现加电场将使管端局域态密度图中的空态能级向费米能级以下移动. 基于本文的电子态密度计算可预期碳纳米管顶端掺 Si 有利于场致电子发射特性.
\end{abstract}

关键词 碳纳米管 掺杂 电子结构 场发射

碳纳米管自问世以来 ${ }^{[1]}$, 一直是科技研究的热点 之一. 其良好的机械、力学及电学性能使它在多方面 得到应用. 1995 年Heer等人 ${ }^{[2]}$ 利用场致发射原理制成 碳纳米管电子枪, 其良好的场发射性质受到人们的 普遍重视. 许多实验表明, 碳纳米管由于化学稳定性 较强, 机械强度较高, 具有比较高的形貌比 (aspect ratio), 在场发射方面有着很好的应用前景, 人们一 直在寻找着更好利用碳纳米管进行场发射应用的方 法. 早在 1999 年就已经有封装好的小型碳纳米管场 发射显示器问世 ${ }^{[3]}$. Gao等人 ${ }^{[4]}$ 对碳纳米管阵列进行 了石墨化, 发现可以提高场发射电流, Chen等人 ${ }^{[5]}$ 从 实验上测定了碳纳米管的排列方向与场发射的关系, $\mathrm{GaO}$ 等人 ${ }^{[6]}$ 用电子显微技术测定了多壁碳纳米管尖端 的场发射功函数.

另一方面, 为充分利用碳纳米管良好的场致发 射特性, 有关其特性的理论研究也在进一步进行. 1997 年Carroll等人[7]通过实验和计算提出, 碳纳米管 的尖端拓扑结构是决定其局域性质的关键. Buldum和 $\mathrm{Lu}^{[8]}$ 提出, 顶端封闭碳纳米管的场发射性能比较优 越，Kim等人 ${ }^{[9]}$ 讨论了在电场下封闭碳纳米管的态密 度变化对于场发射的影响; Park等人 ${ }^{[10]}$ 的计算表明在 碳纳米管顶端掺氧将提高场发射性能, Akdim等人 用DFT理论研究了 $\mathrm{O}_{2}$ 的吸附对于场发射的影响, Maiti等人 ${ }^{[12]}$ 从理论上验证了实验上发现的碳纳米管 顶端吸附水会提高场发射电流的现象, Zhou等人 ${ }^{[13,14]}$ 从理论上提出碳纳米管顶端HOMO-LUMO能级间的 gap与场发射性质联系密切, Zhang等人 ${ }^{[15]}$ 对碳纳米管 的尖端进行了 $\mathrm{B}, \mathrm{N}$ 的取代掺杂计算, 研究了它们引 入的掺杂态对于场发射的影响. 人们通常用传统的
F-N方程来研究碳纳米管的场发射 ${ }^{[16]}$; Bonard 等 人 ${ }^{[17]}$ 通过实验发现单个碳纳米管在低电压下符 合 F-N规则. 但碳纳米管的发射性质与金属场致发 射 器件不同, 并不完全符合F-N方程, 基于量子理论计 算的长碳纳米管场发射研究表明存在一种新机制 ${ }^{[18]}$.

最近, 有实验指出在C60 上掺 $\mathrm{Si}$ 获得成功 ${ }^{[19]}$, 也 有相应理论计算结果发表 ${ }^{[20]}$, 此外一些研究者探索 了在碳纳米管壁上掺 $\mathrm{Si}$ 带来的几何及电子结构的变 化 ${ }^{[21 ~ 23]}$. 基于相关研究并考虑 $\mathrm{Si}$ 与 $\mathrm{C}$ 的电子组态及相 关的成键性以及 SP3 或SP2 的杂化方式, 我们认为 Si 替换纳米管尖端的 C原子有可能在电子态上出现令 人感兴趣的结果. 由于 $\mathrm{Si}$ 基底上生长碳纳米管是一种 常用的方法, 因此掺Si研究也有一定的现实意义. 本 文选择Si作为杂质进行碳纳米管顶端的替代式掺杂, 计算其几何结构及电子结构的变化, 并且讨论掺杂 对于碳纳米管尖端场发射性质的影响. F-N方程指出, 与场发射直接相关并可以调节的两个参量是功函数 $\varphi$ 和放大系数 $\beta$. 其中功函数 $\varphi$ 由体系的电子结构决 定; 而放大系数 $\beta$ 则与体系的几何形貌密切相关. 参 考F-N理论的基本思想, 本文将着重分析与电子结构 及几何形貌相关的参量, 研究掺Si对碳纳米管尖端场 发射性能的影响.

\section{1 计算方法及模型}

我们选择两种最常见的顶端封闭金属型 $((5,5)$ 型 和 $(9,0)$ 型)碳纳米管进行掺杂研究. 并采用以密度泛 函理论(DFT)为基础的DMol量子化学程序 ${ }^{[24]}$ 进行计 算. 交换关联势选择GGA近似下的BLYP形式 ${ }^{[25,26]}$, 基组采用DND基组. 为获得掺杂体系的基态构型, 
我们对所有的体系进行了几何结构优化, 优化过程 中弛豫了碳管顶端的 $\mathrm{C}$ 原子及 $\mathrm{Si}$ 原子, 而固定其余 $\mathrm{C}$ 原子, 当能量梯度小于 $10^{-5} \mathrm{Ha}$ 时, 我们认为体系已 达到其基态构型.

对于(5,5)型纳米管(图 1(a)), 我们计算了管壁和 封闭端共 150 个碳原子的体系, 开口端用氢饱和以消 除长宽比及开口端悬挂键对电子态的影响. 在尖端 进行了 4 种模型掺杂计算, 包括掺入一个Si于不同位 置(3 种掺杂方式, 分别置于 I 最顶端五元环, II 第二 原子层, III第三原子层), 以及在第二原子层的六元 环上两个对角位置以 $\mathrm{Si}$ 替换 C原子(第IV 种掺杂方式), $\mathrm{Si}$ 的位置选取对应于掺杂 $\mathrm{C}_{60}$ 的最稳定结构 ${ }^{[20]}$.

对于(9,0)型纳米管(图 1(b)), 我们选取了管壁和 尖端共 183 碳原子体系, 开口端用氢封闭, 对最顶端 六元环上的 C 原子进行了单 $\mathrm{Si}$ (第 $\mathrm{V}$ 种掺杂方式)和双 $\mathrm{Si}$ (第 VI种掺杂方式)的取代, 掺两个 Si 原子时仍采用 对角掺杂.

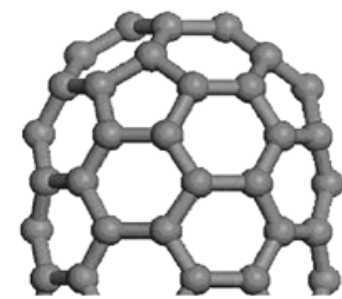

(a)

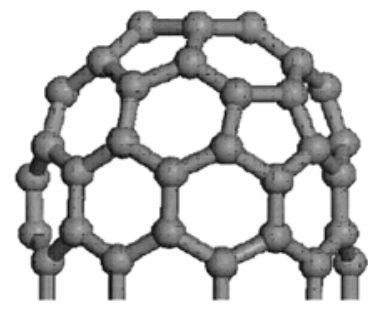

(b)
图 1 计算中所用碳管模型图

(a) 完整 $(5,5)$ 型碳纳米管, (b) 完整 $(9,0)$ 型碳纳米管

\section{2 结果及讨论}

\section{1 能量学计算}

为讨论各个体系的稳定情况, 我们定义掺 Si 体 系的形成能为

$$
E_{\text {form }}=\left(E_{\text {doping }}-E_{\text {pure }}\right)-\left(E_{\mathrm{Si}}-E_{\mathrm{C}}\right),
$$

其中 $E_{\text {pure }}$ 与 $E_{\text {doping }}$ 分别为掺杂前后体系的总能, $E_{\mathrm{C}}$ 与 $E_{\mathrm{Si}}$ 分别为两种原子的化学势(经计算, $C$ 原子的化学 势是-38.08 $\mathrm{Ha}$, 而每个 $\mathrm{Si}$ 原子的化学势为-289.49 $\mathrm{Ha}), E_{\mathrm{Si}}$ 由计算 $\mathrm{Si}$ 块体材料总能对原子数平均得到, 而 $E_{\mathrm{C}}$ 则由计算碳管总能对原子数平均得到. 计算结 果列于表 1 . 可以看出, 对 $(5,5)$ 型碳管而言, Si 在最顶
层取代 C 原子时形成能最低, 而随着占位位置远离 尖端, $E_{\text {form }}$ 逐渐增加. 此外, 第二个 $\mathrm{Si}$ 原子的占位使

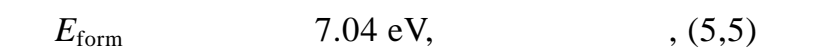
管更倾向于在尖端形成单格点的 Si 杂质占位, 而高 浓度的杂质取代在 $(5,5)$ 型碳管中在能量上是不利的. 而 $(9,0)$ 型碳管则不同, 在最顶端已经有一个 Si 原子 的情况下再掺入第二个 $\mathrm{Si}$ 原子, $E_{\text {form }}$ 仅从 $1.24 \mathrm{eV}$ 上 升至 $1.68 \mathrm{eV}$. 而且优化的结果也表明, $(9,0)$ 碳管结构 仍然比较稳定. 因此可以预期, 高浓度的 Si 掺杂在 $(9,0)$ 型碳管上更易实现. 与此同时, 这个结果也表明, 碳管的手性对于杂质浓度有很明显的调制作用.

根据稳定性分析, 我们选择了 I, IV, V, VI几种 掺杂方式进行研究, 其基态构型如图 2, 并对杂质附近 的原子间键长进行了分析, 结果列于表 2. 可以看到, Si 与最近邻的 $\mathrm{C}$ 形成较 $\mathrm{C}-\mathrm{C}$ 键更长的 $\mathrm{Si}-\mathrm{C}$ 键. 此外, 对于 $(5,5)$ 型碳管, C-C 键几乎普遍增大了 $0.04 \AA$, 而对 于 $(9,0)$ 型的碳管, C-C 键几乎不受 Si 掺杂的影响, 因 此在 $(9,0)$ 型碳管中双 $S i$ 占位引起的畸变更小, 这也解 释了双 Si 占位在 $(9,0)$ 型碳管中更容易实现的成因.

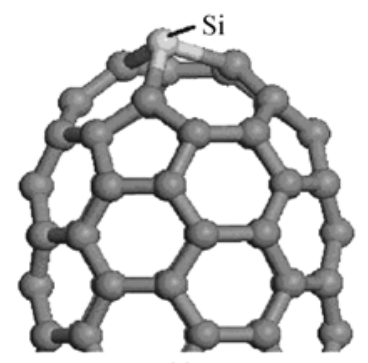

(a)

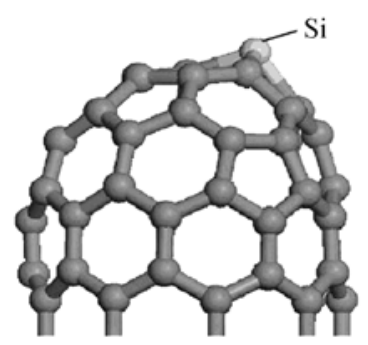

(c)

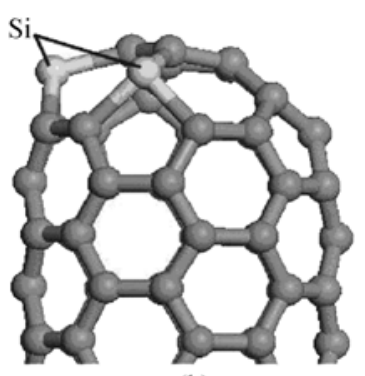

(b)

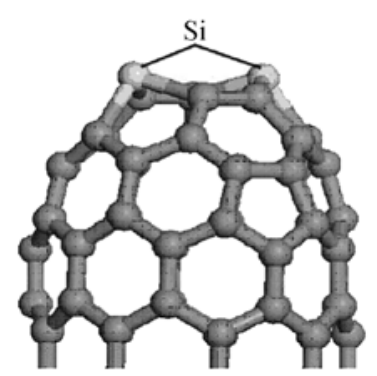

(d)
图 2 碳纳米管顶端掺 Si 后的稳定结构模型 (a) $(5,5)$ 型碳纳米管顶端掺杂一个 Si, (b) $(5,5)$ 型碳纳米管顶端掺杂两个 $\mathrm{Si}$, (c) $(9,0)$ 型碳纳米管顶端掺杂一个 $\mathrm{Si}$, (d) $(9,0)$ 型碳纳米管顶端掺杂两个 $\mathrm{Si}$

表 1 各掺杂情况形成能对比

\begin{tabular}{cccccc}
\hline 构型 & $(5,5)$ 管掺 $1 \mathrm{Si}$ 于第一层 $(5,5)$ 管掺 $1 \mathrm{Si}$ 于第二层 $(5,5)$ 管掺 $1 \mathrm{Si}$ 于第三层 $(5,5)$ 管掺 $2 \mathrm{Si}$ 于第二层 $(9,0)$ 管掺 $1 \mathrm{Si}$ 于第一层 $(9,0)$ 管掺 2Si 于第一层 \\
\hline 形成能 $/ \mathrm{eV}$ & 1.19 & 1.26 & 1.47 & 7.04 & 1.24 \\
\hline
\end{tabular}


表 2 掺杂前后 $(5,5)$ 型及 $(9,0)$ 型碳纳米管顶端键长的变化值

\begin{tabular}{ccccccc}
\hline & 未掺杂(5,5)CNT & 1Si 掺杂(5,5)CNT & 2Si 掺杂(5,5)CNT & 未掺杂(9,0)CNT & 1Si 掺杂(9,0)CNT & 2Si 掺杂(9,0)CNT \\
\hline C一C p-h 键长/ $\AA$ & 1.45 & $1.45 ~ 1.49$ & $1.43 \sim 1.47$ & $1.45 ~ 1.46$ & $1.45 \sim 1.47$ & $1.45 \sim 1.46$ \\
C一C h-h 键长 $/ \AA$ & 1.37 & $1.41 \sim 1.42$ & $1.40 \sim 1.42$ & $1.39 \sim 1.41$ & $1.39 \sim 1.43$ & 1.40 \\
$\mathrm{Si}-\mathrm{C}$ p-h 键长 $/ \AA$ & - & 1.86 & $1.85 \sim 1.89$ & - & $1.85 \sim 1.86$ & $1.86 \sim 1.88$ \\
$\mathrm{Si}$-C h-h 键长 $/ \AA$ & - & 1.81 & 1.84 & - & 1.82 & 1.85 \\
\hline
\end{tabular}

a) p-h 为五元环和六元环共有边, h-h 为两个六元环共有边

键长的变化导致了几何形状的改变, Si原子将伸 展在碳笼的外面(图 2). 而不同的几何形状会导致场 发射放大因子 $\beta$ 有差别, Dyke等人 ${ }^{[27]}$ 证明了放大 因子

$$
\beta=1 / \alpha \gamma,
$$

其中 $\alpha$ 为常数, $\gamma$ 为发射器件尖端的曲率半径, 在掺 杂情况下, 尖端形状发生改变, 产生一个凸起, 导致 曲率半径变小, 可认为这种变化将使 $\beta$ 增大. 虽然现 在普遍认为碳纳米管的场致发射性质在高电压下与 F-N 方程不完全符合, 但仍可视为是定性研究的重要 参考. 考虑到管端变尖有利于电荷积聚, 从而从几何 结构角度说明了加入 Si 对场发射的可能增强作用.

\section{2 电子结构计算}

对于掺杂前后的成键极性, 我们从差分电荷密 度图(图 3)可知, Si 取代 C 以后, Si-C 键与 $\mathrm{C}-\mathrm{C}$ 键 相比有着很强的极性. 从键序(bond order)的计算可 以看出, $\mathrm{Si}-\mathrm{C}$ 键强(键序 $0.3 \sim 0.4$ )弱于 $\mathrm{C}-\mathrm{C}$ 键(键序 1.1 1.2).

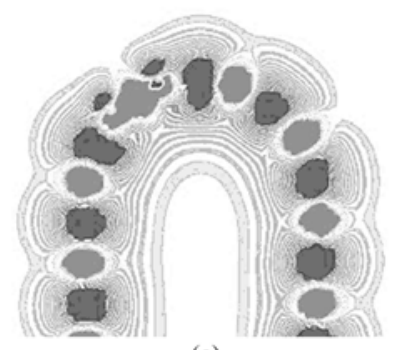

(a)

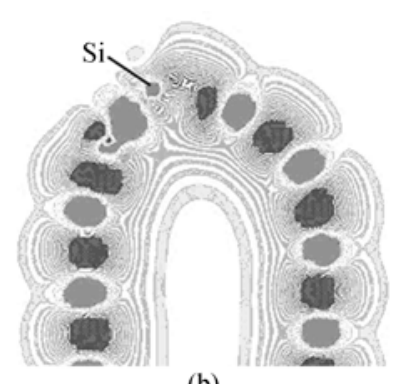

(b)
强, 而反键态峰出现在 $E_{\mathrm{F}}$ (费米能级)以上 $0.8 \mathrm{eV}$ 处. 随着 $\mathrm{Si}$ 杂质在尖端取代C, 总局域态密度的噟能隙有 所减小，因此其金属性逐渐增强. 此外，反键态的主 要成分由Si原子贡献, 距离 $E_{\mathrm{F}}$ 仅有 $0.15 \mathrm{eV}$ 左右, 且 $E_{\mathrm{F}}$ 处的态密度有比较明显的提高. 费米能级附近的高 局域态将使碳纳米管产生更好的场致发射性能 ${ }^{[9]}$, 因 此从DOS分析中可以看到, 单格点 $S i$ 掺杂对于 $(5,5)$ 型 碳管的场致发射性能有所改善. 从图 5(c)和(d)中也 可看到, Si的成键呈现出各向异性的特征, 主要是由 于Si与尖端第二层C原子发生轨道杂化导致. 图 6 表 明, 随着Si浓度的增加, 碳管的金属性进一步增强, 且反键态峰的位置几乎与 $E_{\mathrm{F}}$ 重合. 因此, 高浓度的 $\mathrm{Si}$ 掺杂确实有助于改善碳管的场致发射性能.

由图 7 9 可看到, $(9,0)$ 型碳管显示出了类似的特 点, 惟一不同之处在于单个 $\mathrm{Si}$ 原子尖端替位时, 与其 成键较强的是尖端第一层的 C 原子, 与 $(5,5)$ 型碳管情 况正好相反, 这表明了碳管的手性对于掺杂成键的 影响. 特别需要注意的是, 因为对于 $(9,0)$ 型碳管, 双 Si 占位的 $E_{\text {form }}$ 并不高, 所以高浓度 Si 掺杂的 $(9,0)$ 型 碳管可以预计有较强的应用价值和前途.

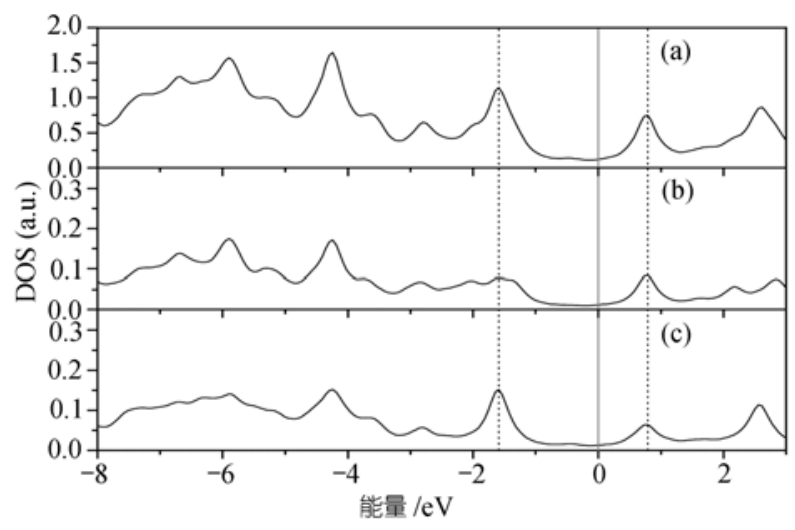

图 $4(5,5)$ 型碳纳米管掺杂前的尖端局域总态密度与各原 子上的局域态密度

(a) 总局域态密度, (b) 顶端五元环上 C 的局域态密度, (c) 第二层 C 上的局域态密度 


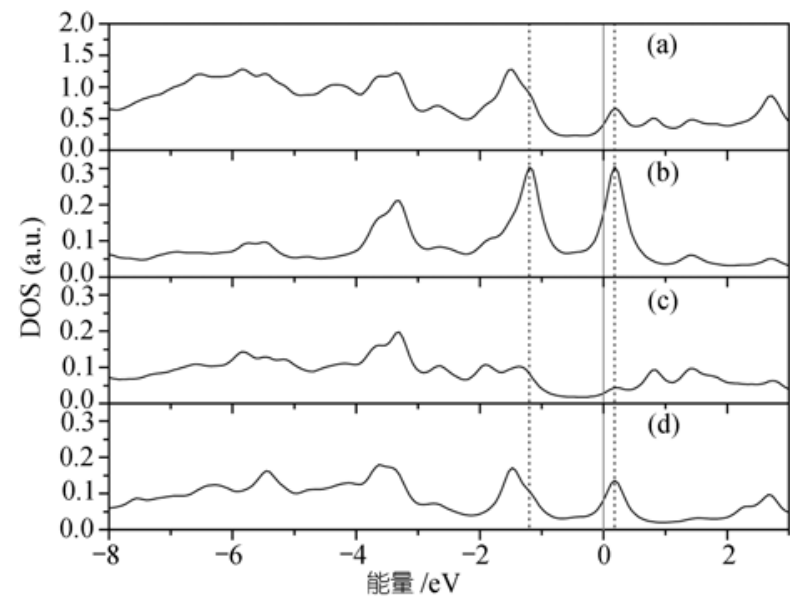

图 $5(5,5)$ 型碳纳米管尖端掺一个 Si 之后的尖端局域总态 密度和各原子的局域态密度

(a) 总局域态密度, (b) 顶端五元环上 $\mathrm{Si}$ 的局域态密度, (c) 顶端五元 环上与 $\mathrm{Si}$ 第一近邻的 C 局域态密度, (d) 第二层上与 $\mathrm{Si}$ 第一近邻 C 的 局域态密度

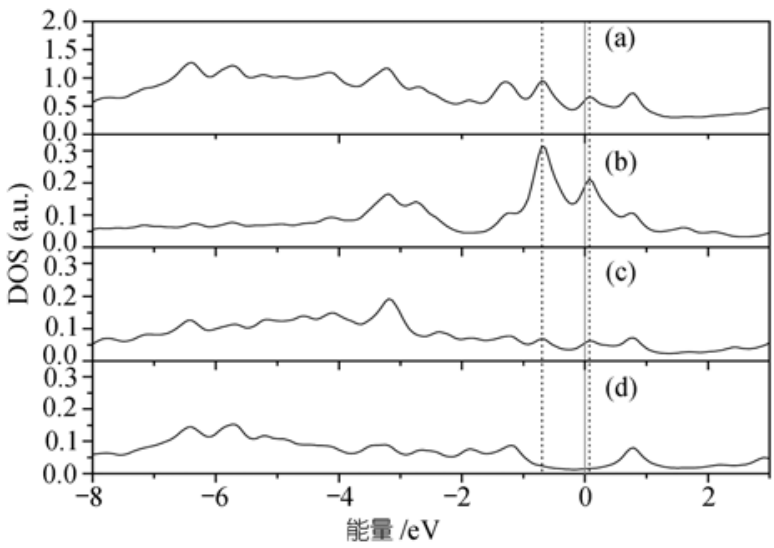

图 $6(5,5)$ 型碳纳米管尖端掺两个 Si 之后的尖端局域总态 密度和各原子的局域态密度

(a) 总局域态密度, (b) 第二层六元环上 $\mathrm{Si}$ 的局域态密度, (c) 顶端五 元环上与 Si 第一近邻的 C 局域态密度, (d) 顶端五元环上与 $\mathrm{Si}$ 第二近 邻 C 的局域态密度

对于加入电场的情况, 我们研究了电场对于碳 纳米管顶端局域态密度的影响. 我们选择了两种强 度的电场 $(0.5 \mathrm{~V} / \AA$ 和 $1 \mathrm{~V} / \AA)$ 加在碳纳米管上. 计算发 现(图 10 和 11), 电场的加入使局域态密度整体向低 能端移动, 相应的反键态的峰位置更加靠近 $E_{\mathrm{F}}$, 因此 电子占据反键态的可能性更大. 当加入 $1 \mathrm{~V} / \AA ̊$ 的电场 时, 可以看到未掺杂 $(5,5)$ 型碳管顶端局域总态密度 的峰由费米能级以上 $0.8 \mathrm{eV}$ 移到费米能级处, 从而增 大了费米能级处电子的态密度, 与Kim ${ }^{[9]}$ 提到的发射 场发射状态相符合，当电场强度为 $0.5 \mathrm{~V} / \AA$ 时, 我

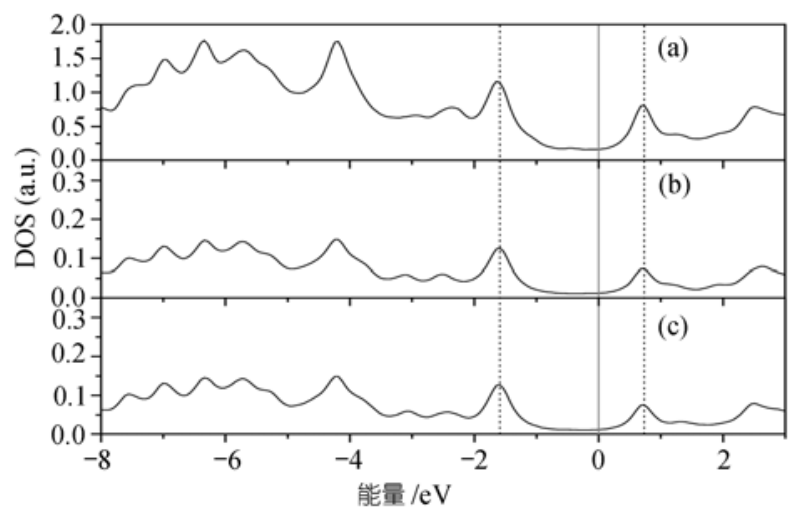

图 $7(9,0)$ 型碳纳米管掺杂前的尖端局域总态密度与各原 子上的局域态密度

(a) 总局域态密度, (b) 顶端六元环上 C 的局域态密度, (c) 第二层 C 上的局域态密度

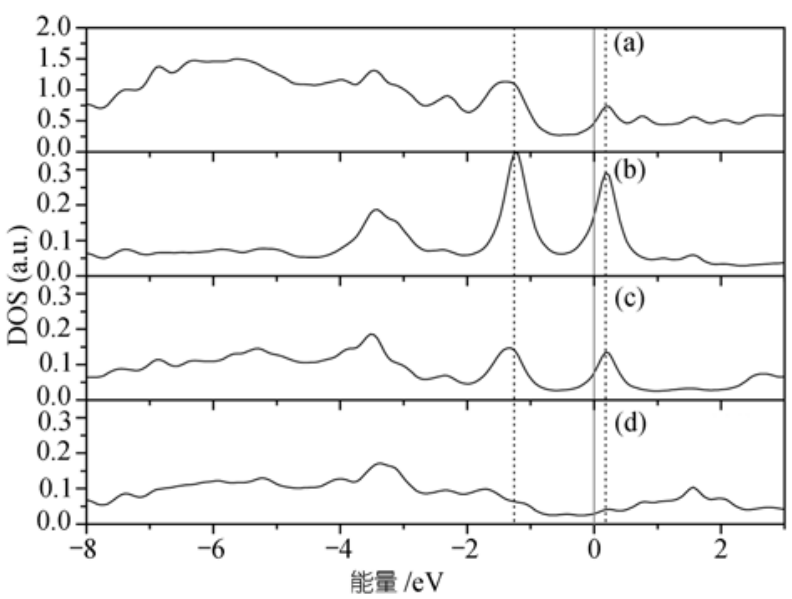

图 $8(9,0)$ 型碳纳米管尖端掺一个 Si 之后的尖端局域总态 密度和各原子的局域态密度

(a) 总局域态密度, (b) 顶端六元环上 Si 的局域态密度, (c) 顶端六元 环上与 $\mathrm{Si}$ 第一近邻的 C 局域态密度, (d) 第二层上与 $\mathrm{Si}$ 第一近邻 C 的 局域态密度

们发现掺杂的碳纳米管比未掺杂碳纳米管更快的在 费米能级处产生局域态密度峰, 因此推测可能在较 小电场强度下产生场发射现象.

我们定义电子发射到无穷远点的能量值 (最高占 据态 HOMO 的绝对值)为功函数, 各种体系的 HOMO 列于表 3 和 4. 可以看到, 掺杂前后 HOMO 的变化仅 为 $0.03 \sim 0.04 \mathrm{eV}$, 但电子在电场作用下也可能通过 “过渡” 形式发射, 即以最低非占据态(LUMO)为跃 迁阶梯进行发射, 而掺 Si 对 LUMO 能级的改变则是 $10^{-1} \mathrm{eV}$ 量级, 因此可以预计, Si 对于体系 LUMO 能 级的调控会明显地影响碳纳米管场发射的物理效应. 


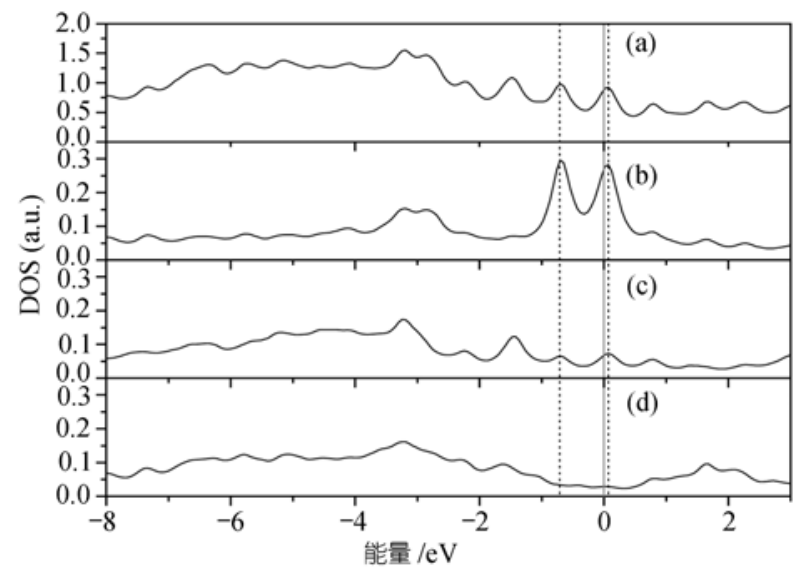

图 $9(9,0)$ 型碳纳米管尖端掺两个 Si 之后的尖端局域总态 密度和各原子的局域态密度

(a) 总局域态密度, (b) 顶端六元环上 Si 的局域态密度, (c) 顶端六元 环上与 Si 第一近邻的 C 局域态密度, (d) 第二层上与 Si 第二近邻 C 的 局域态密度

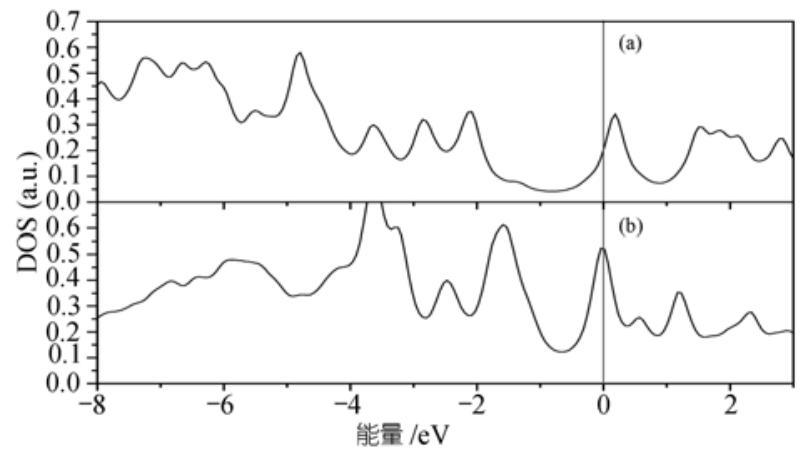

图 10 掺杂前后 $(5,5)$ 型碳纳米管顶端局域电子态密度(外 场 $0.5 \mathrm{~V} / \AA$ )

(a) 掺杂前的局域态密度, (b) 掺一个 Si 后 $\mathrm{Si}$ 及周围 C 原子的局域态密度

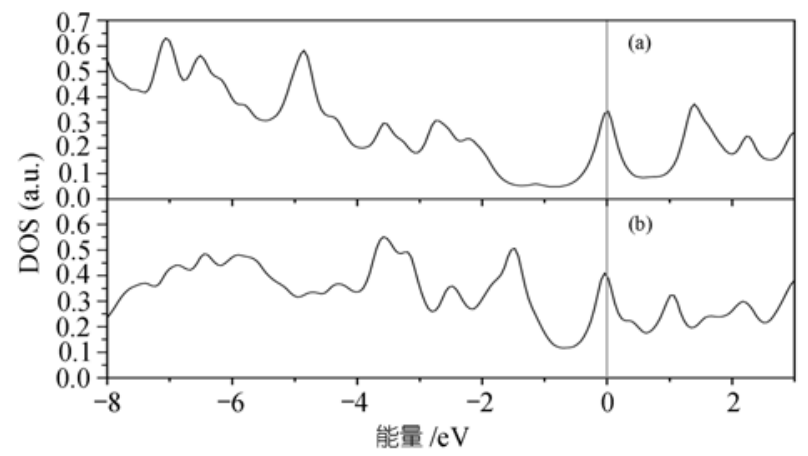

图 11 掺杂前后 $(5,5)$ 型碳纳米管顶端局域电子态密度 (外场 $1 \mathrm{~V} / \AA$ )

(a) 掺杂前的局域态密度, (b) 掺一个 Si 后 Si 及周围 C 原子的局域态密度
表 $3(5,5)$ 型碳纳米管各种掺杂的 HOMO, LUMO, $E_{\text {eff }}$ (有 效功函数)及其变化值

\begin{tabular}{lccc}
\hline \multicolumn{1}{c}{ 构型 } & \multirow{2}{*}{$(5,5)$ 型纯碳管 } & $\begin{array}{c}\text { 掺 } 1 \mathrm{Si} \text { 的 }(5,5) \\
\text { 型碳管 }\end{array}$ & $\begin{array}{c}\text { 掺 } 2 \mathrm{Si} \text { 的 }(5,5) \\
\text { 型碳管 }\end{array}$ \\
\hline $\mathrm{HOMO} / \mathrm{eV}$ & -4.745 & -4.708 & -4.715 \\
$\mathrm{LUMO} / \mathrm{eV}$ & -3.936 & -4.124 & -4.229 \\
$E_{\mathrm{eff}} / \mathrm{eV}$ & 0.405 & 0.292 & 0.243 \\
$E_{\mathrm{eff}}$ 降低 $/ \mathrm{eV}$ & 0 & 0.113 & 0.162 \\
\hline
\end{tabular}

表 $4(9,0)$ 型碳纳米管各种掺杂的 HOMO, LUMO, $E_{\mathrm{eff}}$ 及其变化值

\begin{tabular}{lccc}
\hline \multicolumn{1}{c}{ 构型 } & $(9,0)$ 型纯碳管 & $\begin{array}{c}\text { 掺 1Si 的 }(9,0) \\
\text { 型碳管 }\end{array}$ & $\begin{array}{c}\text { 掺 2Si 的 }(9,0) \\
\text { 型碳管 }\end{array}$ \\
\hline $\mathrm{HOMO} / \mathrm{eV}$ & -4.284 & -4.264 & -4.302 \\
$\mathrm{LUMO} / \mathrm{eV}$ & -4.019 & -4.075 & -4.219 \\
$E_{\mathrm{eff}} / \mathrm{eV}$ & 0.133 & 0.095 & 0.042 \\
$E_{\mathrm{eff}}$ 降低/eV & 0 & 0.038 & 0.091 \\
\hline
\end{tabular}

基于上述考虑，我们引入有效功函数 $\left(E_{\text {eff }}\right)$ 的概 念, 计 LUMO 到 Fermi 的能级的差为有效功函数, 结 果表明(表 3 和 4), 掺一个 $\mathrm{Si}$ 的(5,5)型纳米管的 $E_{\text {eff }}$ 将减小 $0.11 \mathrm{eV}$ 左右(相对于未掺杂); 而掺杂了两个 $\mathrm{Si}$ 的 $(5,5)$ 型纳米管 $E_{\mathrm{eff}}$ 减小了 $0.16 \mathrm{eV}$. 这也说明了加 入 $\mathrm{Si}$ 将减小 $E_{\text {eff }}$ 值, 有利于场发射.

HOMO 及 LUMO 轨道电子密度分布示于图 12 和 13, 易见 LUMO 主要集中在 Si 原子上, 即体系的 LUMO 主要为 Si 所贡献, 随着 Si 浓度的增加, 可以 看到 LUMO 越来越集中于碳管的尖端, 而 HOMO 分 布并未发生明显的变化, 与表 3 的结果相一致.

费米能级处的态密度主要由 HOMO/LUMO 轨道 所贡献, 掺 Si 后这两个轨道主要由 Si 原子的轨道组 成(图 12 和 13), 而从 DOS 图(图 5 9)中可以看到, Si 原子的这些轨道主要占据在费米能级处, 从而增加 了费米能级上的态密度. 而 Si 的电子亲和势(electron affinity)(133.6 kJ/mol)小于 C $(153.9 \mathrm{~kJ} / \mathrm{mol})^{1)}$, 因此 $\mathrm{Si}$ 原子上处于费米能级处的电子在电场下更易发射, 此外, 费米能级处态密度的增大对应着可发射电子 数的增多, 由此我们认为掺 $\mathrm{Si}$ 的碳管场发射功函数 将会相应下降. 上面的有效功函数计算也支持了这 个结果.

从图 12 和 13 中, 结合以上态密度的分析, 可以 看出几种掺杂方式中发射性能最好的是顶端六元环 中掺两个 Si 的 $(9,0)$ 型碳纳米管, 而这种掺杂会通过 增强“过渡”发射形式的方式完成对碳纳米管场致发 


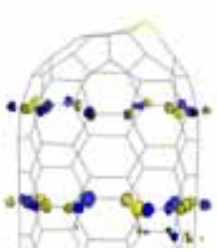

(a)

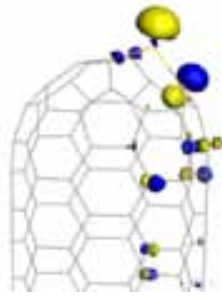

(b)

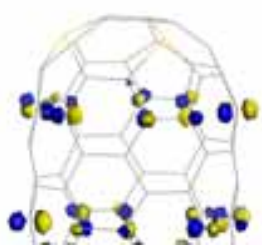

(c)

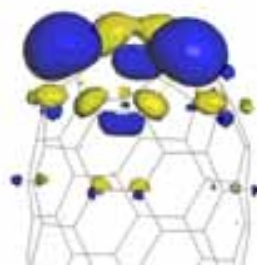

(d)

图 12 掺杂后的 $(5,5)$ 型碳纳米管 HOMO/LUMO 轨道电子密度分布图

(a) 单个 Si 掺杂 $(5,5)$ 碳管的 HOMO 分布, (b) 单个 Si 掺杂 $(5,5)$ 碳管的 LUMO 分布, (c) 两个 Si 掺杂 $(5,5)$ 碳管的 HOMO 分布,

(d) 两个 Si 掺杂 $(5,5)$ 碳管的 LUMO 分布

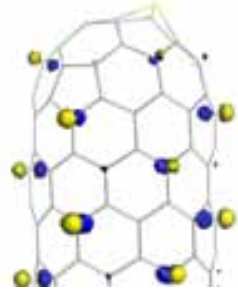

(a)

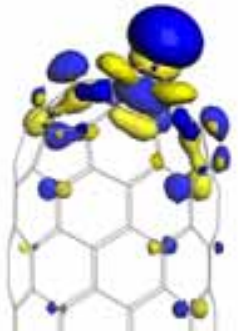

(b)

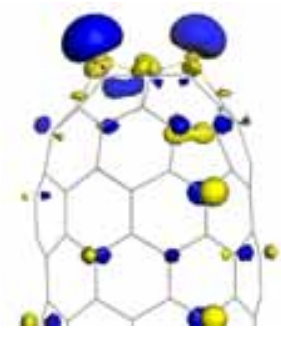

(c)

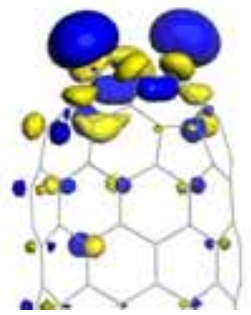

(d)

图 13 掺杂后的 $(9,0)$ 型碳纳米管 HOMO/LUMO 轨道电子密度分布图

(a) 单个 Si 掺杂 $(9,0)$ 碳管的 HOMO 分布, (b) 单个 Si 掺杂( $(9,0)$ 碳管的 LUMO 分布, (c) 两个 Si 掺杂 $(9,0)$ 碳管的 HOMO 分布,

(d) 两个 Si 掺杂 $(9,0)$ 碳管的 LUMO 分布

\section{射性能的改善.}

\section{3 结论}

两种金属型碳纳米管 $((5,5)$ 和 $(9,0))$ 的封闭端掺 $\mathrm{Si}$ 前后几何结构变化及局域电子态的计算发现：从几 何结构看, 掺 Si使得碳纳米管尖端变得尖锐, 导致管 端容易积聚电荷，而有利于电子的发射; 从电子结构 看, 掺 Si 使得尖端出现了很强的掺杂态, 使费米能级 处的电子态密度大大加强, 并导致功函数下降, 而加 强场发射效应; 并且，外场将使碳纳米管尖端处的空 态能级向费米能级以下移动. 本文第一原理计算表 明，碳纳米管尖端掺 Si 有可能提高其场发射性能.

致谢 作者之一得到了周刚、蔡军、严家安、陈征征等的 热心帮助, 在此表示衷心感谢. 本工作为国家自然科学基 金(批准号: 90101004)资助项目.

\section{参考文献}

1 Iijima S. Helical microtubules of graphitic carbon. Nature (London), 1991, 354: 56 58[DOI]

2 de Heer W A, Châtelain A, Ugarte $\mathrm{B}$ : $/ \mathrm{A}$ carbon nanotube field-emission electron source. Science, 1995, 270: 1179 1180

3 Choi W B, Chung D S, Kang J H, et al. Fully sealed, high-brightness carbon-nanotube field-emission display. Applied Physics Letters, 1999, 75 (20): 3129 3131[DOI]

4 Gao Han, Mu Chen, Wang Fan, et al. Field emission of large-area and graphitized carbon nanotube array on anodic aluminum oxide template. Journal of Applied Physics, 2003, 93(9): 5602 5605[DOI]

5 Chen Yan, Shaw D T, Guo Liping. Field emission of different oriented carbon nanotubes. Applied Physics Letters, 2000, 76(17): 2469 2471[DOI]

6 Gao Ruping, Pan Zhengwei, Wang Zhong L. Work function at the tips of multiwalled carbon nanotubes. Applied Physics Letters, 2001, 78(12): 1757 1759[DOI]

7 Carroll D L, Redlich P, Ajayan P M, et al. Electronic structure and localized states at carbon nanotube tips. Phys Rev Lett, 1997, 78: 2811 2814[DOI]

8 Buldum A, Lu Jianping. Electron field emission properties of closed carbon nanotubes. Phys Rev Lett, 2003, 91: 236801 236804[DOI]

9 Kim C, Kim B, Lee S M, et al. Electronic structures of capped carbon nanotubes under electric fields. Phys Rev B, 2002, 65: 165418 165423[DOI] 
10 Park N, Han S, Ihm J. Effects of oxygen adsorption on carbon nanotube field emitters. Phys Rev B, 2001, 64: 125401 165404[DOI]

11 Akdim B, Duan Xiaofeng, Pachter R. The effects of $\mathrm{O}_{2}$ adsorbates on field emission properties of single-wall carbon nanotubes. Nano Letters, 2003, 3(9): 1209 1214 [DOI]

12 Maiti A, Andzelm J, Tanpipat N. Effect of adsorbates on field emission from carbon nanotubes. Phys Rev Lett, 2001, 87: 155502 155505[DOI]

13 Zhou Gang, Kawazoe Y. Localized valence states characteristics and work function of single-walled carbon nanotubes. Phys Rev B, 2001, 65: 155422 155426

14 Zhou Gang, Duan Wenhui, Gu Binglin. Electronic structure and field-emission characteristics of open-ended single-walled carbon nanotubes. Phys Rev Lett, 2001, 87: 095504 095507[DOI]

15 Zhang Gang, Duan Wenhui, Gu Binglin. Effect of substitutional atoms in the tip on field-emission properties of capped carbon nanotubes. Applied Physics Letters, 2002, 80(14): 2589 2591[DOI]

16 Temple D. Recent progress in field emitter array development for high performance applications. Materials Science and Engineering, 1999, R24: 185 239

17 Bonard J, Dean K A, Coll B F, et al. Field emission of individual carbon nanotubes in the scanning electron microscope. Phys Rev Lett, 2002 , 89: 197602 197605[DOI]

18 Zheng Xiao, Chen GuanHua, Li Zhibing, et al. Quantum-mechanical investigation of field-emission mechanism of a micrometer-long single-walled carbon nanotube. Phys Rev Lett, 2004, 92: 106803
106806[DOI]

19 Fye J L, Jarrold M F. Structures of silicon-doped carbon clusters. J Phys Chem, 1997, 101: 1836 1840

20 Billas I M L, Massobrio C, Boero M, et al. First principles calculations of Si doped fullerenes: Structural and electronic localization properties in $\mathrm{C}_{59} \mathrm{Si}$ and $\mathrm{C}_{58} \mathrm{Si}_{2}$. J Chem Phys, 1999, 111: 6787 6796[DOI]

21 Baierle R J, Fagan S B, Mota R, et al. Electronic and structural properties of silicon-doped carbon nanotubes. Phys Rev B, 2001, 64: 085413 085416[DOI]

22 Mavrandonakis A, Froudakis G E, Schnell M, et al. From pure carbon to silicon-carbon nanotubes: An ab-initio study. Nano Letters, 2003, 3(11): 1481 1484[DOI]

23 Fagan S B, Mota R, Da Silva A J R, et al. Substitutional Si doping in deformed carbon nanotubes. Nano Letters, 2004, 4(5): 975 977[DOI]

24 Delley B. An all-electron numerical method for solving the local density functional for polyatomic molecules. J Chem Phys, 1990, 92: 508 517[DOI]

25 Becke A D. A multicenter numerical integration scheme for polyatomic molecules. J Chem Phys, 1988, 88: 2547 2553[DOI]

26 Lee C, Yang Weitao, Parr R G. Development of the Colle-Salvetti correlation-energy formula into a functional of the electron density. Phys Rev B, 1988, 37: 785 789[DOI]

27 Dyke W P, Dolan W W. Field emission. Adv Elec El Phys, 1956, 8: 89 93

(2005-03-29 收稿, 2005-07-06 收修改稿) 